\title{
As Dimensões do Arco Elétrico
}

\author{
(Dimensions of the Electric Arc)
}

\begin{abstract}
Louriel O. Vilarinho ${ }^{1}$
${ }^{1}$ Universidade Federal de Uberlândia, Laprosolda - Centro para Pesquisa e Desenvolvimento de Processos de Soldagem, Uberlândial MG,38400-902,vilarinho@mecanica.ufu.br
\end{abstract}

\begin{abstract}
Resumo
Dada a complexidade intrínseca de um arco elétrico utilizado como fonte de energia para a fusão nos processos de soldagem a arco, onde existem gradientes muito elevados de propriedades e material, torna-se difícil estabelecer limites para as dimensões do arco. Em termos práticos esta definição é importante para se estabelecer, por exemplo, preparação de juntas e geometria de peças, de tal forma que interfira de forma adequada na formação do arco. Algumas características podem ser utilizadas para definir as dimensões do arco, como a radiação luminosa (raio óptico) e o campo elétrico (raio elétrico), além da mancha/raiz anódica (peça). Desta forma, é objetivo apresentar e discutir as dimensões do arco elétrico com base nestas características. Espera-se que haja discussão das idéias e resultados aqui apresentados com réplica ao texto escrito a partir da contribuição feita por observações de outros pesquisadores.
\end{abstract}

Palavras-chave: Plasma; Raio óptico; Raio elétrico; Mancha anódica.

\begin{abstract}
The definition of the electric arc dimensions is a complex task, due to its intrinsic complexity, where very high steep gradients of properties and material are found. In practical terms, this definition is important to establish, for instance, joint preparation and workpiece geometry, in such way that they positively interfere on the arc formation. Some characteristics can be used to define the arc dimensions, such as light radiation (optical radius) and the electrical field (electrical radius), besides the anode spot (workpiece). Therefore, it is aimed to present and discuss the electrical arc dimensions basing on these characteristics. It is expected that discussion of the presented ideas and results arises through the reply of other researchers from their own observations.
\end{abstract}

Key-words: Plasma; Optical radius; Electrical radius; Anode spot.

\section{Introdução}

No processo TIG, é fácil estabelecer a distância entre a ponta do eletrodo e a peça, e a esta distância chama-se comprimento (ou altura) do arco. Neste sentido, desconsidera-se a escalada lateral do arco em torno do eletrodo que é função do gás de proteção, material e geometria do eletrodo e da corrente. No processo com eletrodo consumível, o estabelecimento da altura do arco torna-se ainda mais complicado devido aos fenômenos de transferência metálica, onde alguns critérios têm sido estabelecidos na literatura [1]. Entretanto, não apenas a altura do arco é importante, mas também o seu raio, curvatura e dimensões gerais podem levar a diferentes resultados práticos. Por exemplo, em uma junta em $\mathrm{V}$, se o arco tiver maior ou menor raio, o seu campo eletromagnético será influenciado mais ou menos pelo material de base.

Além dos valores geométricos em si, torna-se também importante definir qual a melhor característica para definir um arco elétrico. Dentre elas, destaca-se o nível de radiação

(Recebido em 17/03/2009; Texto Final em 07/08/2009). luminosa, i.e., seu raio óptico, que pode ser visível ou não (UV principalmente) e o campo eletromagnético, i.e., seu raio elétrico. Pode-se ainda estabelecer as dimensões das conexões catódica e anódica, como característica importante para a continuidade do arco e fusão do metal de base. Neste sentido, a Figura 1 traz uma visão esquemática para as dimensões do arco com base nestas características principais, restando ainda estabelecer elementos quantitativos para tal.

Desta forma, as principais dimensões do arco com base nestas características são definidas e valores dimensionais apresentados, onde a importância de cada uma delas é descrita, observando-se a aplicação tecnológica das mesmas. Ressalta-se que características para definição das dimensões do arco têm sido objeto de estudo de longa data [2], com a geração de discussão produtiva sobre o assunto. É justamente neste contexto que se propõe este artigo, como forma de desafio para discussão da comunidade da soldagem.

\section{Valores para as Dimensões do Arco}

Uma das primeiras e mais completas observações das dimensões do arco é apresentada na Ref. [4], onde os valores do raio óptico e mancha/raiz anódica são mostrados na Figura 
2. Dadas as limitações tecnológicas de quando estes resultados foram obtidos, o comprimento de arco utilizado $(10 \mathrm{~mm})$ não se encontra próximo dos valores utilizados na prática. Entretanto, algumas observações qualitativas podem ser feitas com relação aos valores apresentados. Por esta figura também se observa que a largura da poça é menor que o raio óptico do arco. Isto é coerente, se a condução do calor para a peça for levada em consideração.

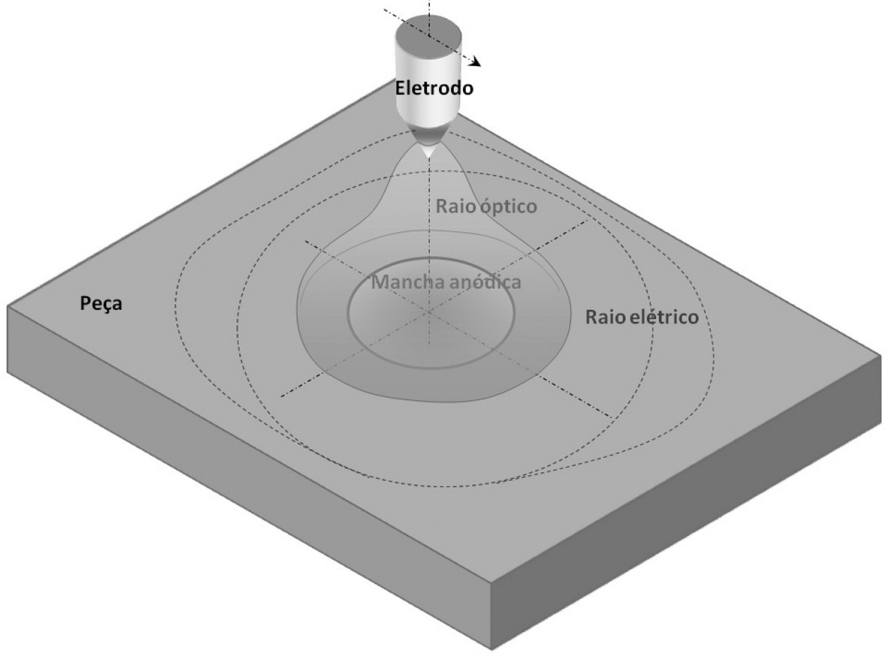

Figura 1. Visão esquemática para as dimensões do arco [3].

Pelos valores de temperatura apresentados, o raio óptico do arco foi definido pelo grau de ionização definido pela curva de densidade de partículas em função da temperatura [5]. Para o caso do argônio, admitindo-se que se a partir da formação de íons de argônio $\left(\mathrm{Ar}^{+}\right)$tem-se arco elétrico, a temperatura seria em torno de $7000 \mathrm{~K}$ (Figura 3). Este valor poderia ser arbitrado em outro nível, como 95\% de ionização. Assim, deve-se estabelecer este nível ao se definir a dimensão do arco com relação ao grau de ionização do mesmo.

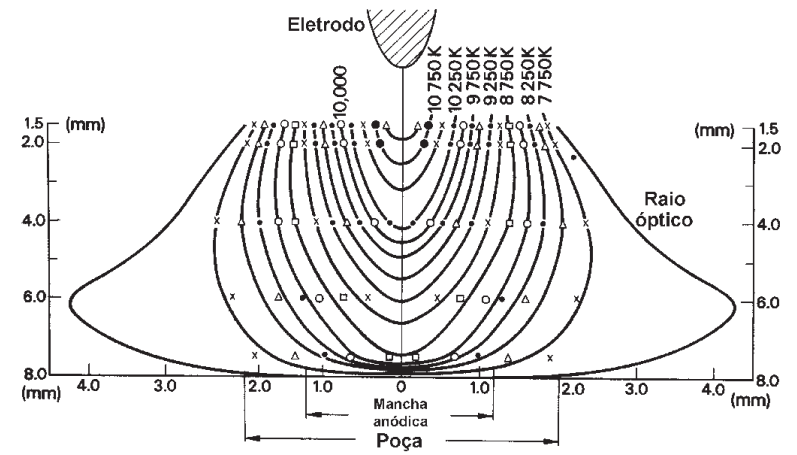

Figura 2. Dimensões propostas para um arco TIG 100 A, argônio puro (Ref. [4], modificado).

Com relação à mancha anódica, esta possui um diâmetro de $\sim 2.5 \mathrm{~mm}$, o que leva a uma densidade de corrente de $\sim 210^{7}$ $\mathrm{A} / \mathrm{m}^{2}$, para a corrente de 100 A utilizada. Este valor está em conformidade com a literatura de soldagem [6]. Deve-se ressaltar que a mancha anódica é menor que a dimensão do arco elétrico próximo ao ânodo. Apesar de esta afirmação parecer

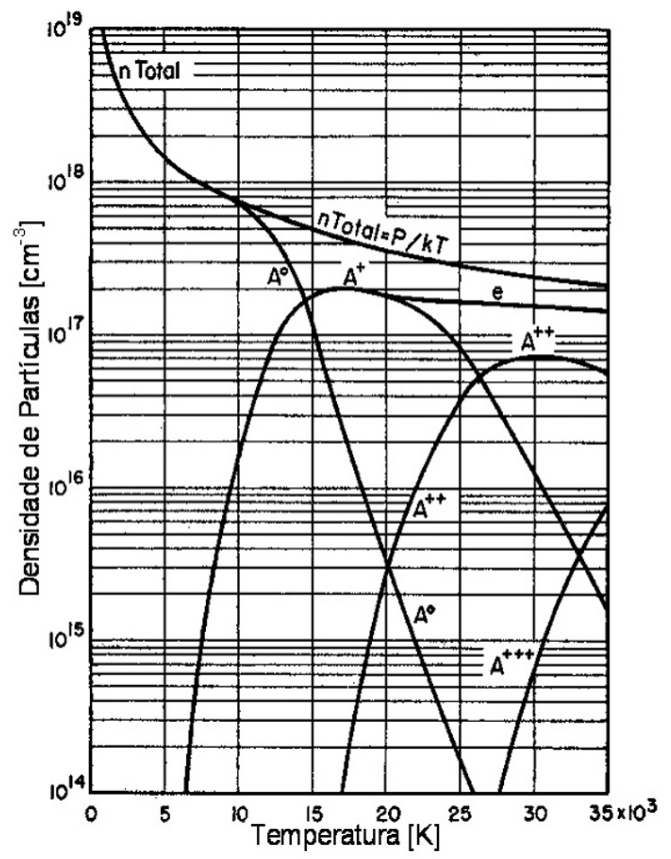

Figura 3. Densidade de partículas em função da temperatura para argônio puro [5], onde $\mathrm{A}^{\circ}$ (ou ArI) é o átomo de argônio neutro excitado, Ar+ (ou ArII) é o íon de argônio monovalente positivo e assim por diante.

contraditória, diferentes autores têm procurado explicar estas observações com base em modelos matemáticos que acoplem ambas as regiões [7].

Neste sentido, com base nas quedas de tensão medidas por diferentes autores e apresentadas na Figura 4 por diferentes autores, estima-se as dimensões do arco próximo ao ânodo (Figura 5). Os valores estimados se baseiam no campo eletromagnético e, como apresentado esquematicamente na Figura 1, são maiores que o raio óptico apresentado na Figura 2. Este raio elétrico representa a posição onde um coletor de eletricidade (sonda de Langmuir) passando através do arco consegue medir o potencial desta posição em condição flutuante (F.C.='floating condition').

Um resultado prático da Figura 5 refere-se ao fenômeno de sopro magnético, ou seja, ao desvio do arco na presença de campo magnético. Embora, o soldador observe o arco de dimensões menores que $5 \mathrm{~mm}$ (raio óptico), a interação com o campo eletromagnético do arco pode ocorrer até $20 \mathrm{~mm}$ ao redor do mesmo.

Como resultados mais recentes, têm-se as dimensões mostradas na Figura 6 para um arco TIG 150 A e argônio puro. Estes valores retratam medições realizadas com uso de sondas de Langmuir [10], da técnica do ânodo bi-partido modificado [9] e filmadora de alta velocidade [3].

A sonda de Langmuir consegue, através de medições de corrente e tensão, estimar a posição onde há a saturação iônica $\left(\mathrm{j}_{\text {isat }}\right)$, a saturação eletrônica $\left(\mathrm{j}_{\mathrm{e}}\right)$ e a condição flutuante (F.C.). Como discutido, a condição flutuante retrata a maior distância a partir do centro onde se consegue medir potencial no plasma. Isto é conseguido pela simples introdução da sonda através do arco e medindo-se o potencial sem aterrá-lo à fonte de 
soldagem (por isto em condição flutuante). Por outro lado, ao se aplicar tensão elétrica na sonda, dependendo-se da polaridade, acontece a saturação iônica (polaridade negativa, pois atrai os íons positivos) e a eletrônica (polaridade positiva, pois atrai os elétrons). Assim, a saturação iônica está relacionada ao balanço entre cargas positivas e a bainha do plasma. Já a saturação eletrônica ocorre onde se anula a bainha de plasma. A bainha do plasma é a princípio formada predominantemente por íons positivos, pois quando se introduz um corpo (sonda) no plasma, estatisticamente, pelo fato dos elétrons terem maior velocidade que os íns positivos, a sonda fica carregada negativamente neste momento inicial. Em seguida, pelo fato de que ao se estabelecer um volume ao redor do plasma, este deve possuir um balanço de cargas próximo de zero, íons positivos circundam a sonda para tentar neutralizar aqueles elétrons que atingiram inicialmente a sonda. Desta forma, se forma uma bainha ao redor da sonda, com cargas predominantemente positivas.

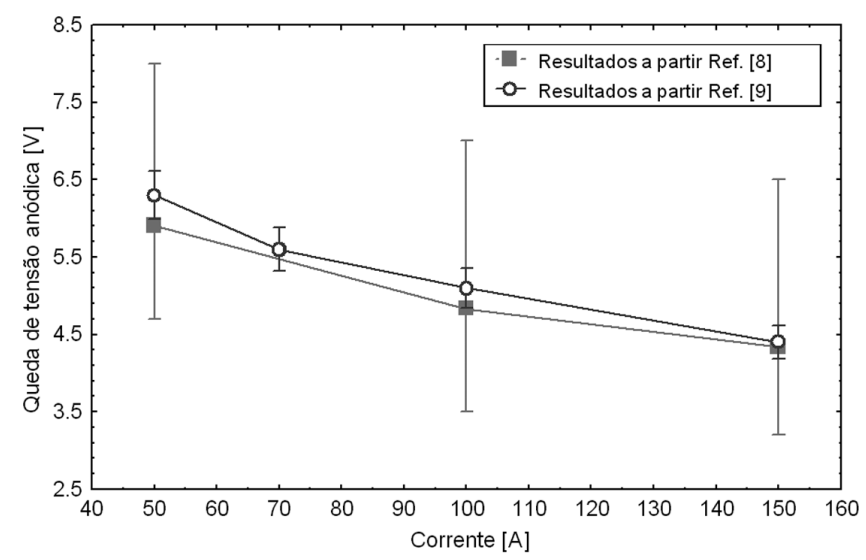

Figura 4. Queda de tensão no ânodo em função da corrente de soldagem $[8,9]$.

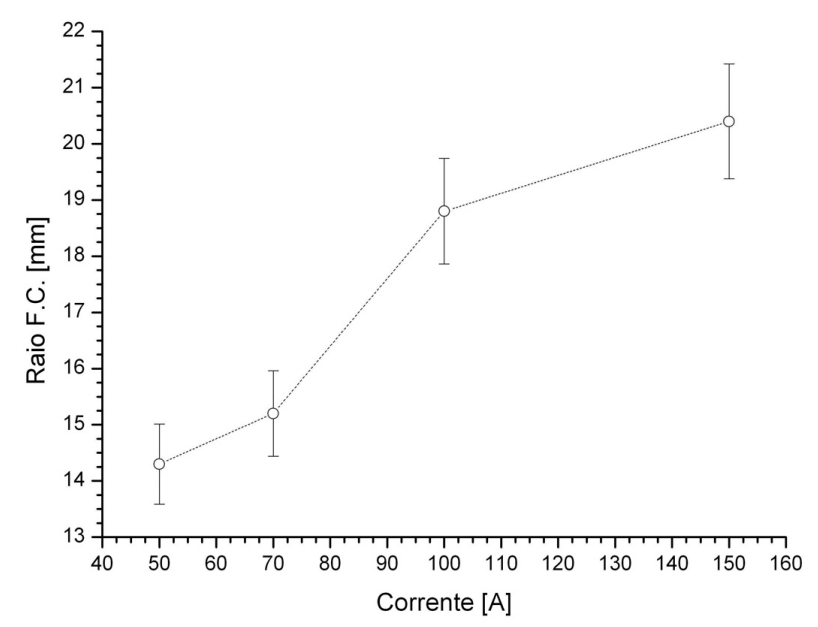

Figura 5. Raio anódico do arco para F.C. [3].

A condição flutuante mede o momento inicial que esta bainha é formada e, portanto, a máxima dimensão onde se caracterizaria o plasma. Ao se aplicar tensão elétrica na sonda altera-se a estrutura da bainha. No caso de saturação iônica se aplicou uma polaridade negativa à sonda, fazendo com que se aumentasse a quantidade de cargas positivas que estão buscando anular o potencial negativo (balanço de cargas). Mas isto tem um limite, pois se aumenta a tensão elétrica, aumenta-se o número de cargas positivas, mas também se aumenta o tamanho da bainha, ou seja, a densidade de íons se mantém (saturação iônica). Raciocínio semelhante ao se aplicar polaridade positiva, onde a bainha começa a diminuir as cargas positivas, passando inclusive a ter mais cargas negativas (elétrons), até o limite onde se passa a ter o balanço de cargas e tamanho da bainha, mantendo-se a densidade de cargas e atingindo a saturação eletrônica.

Assim, justifica-se que a radiação (emissão de fótons em função de alterações eletrônicas nos átomos excitados/íons) ocorra onde se tenha um grande número de elétrons livres. Este ponto seria a saturação iônica, pois as cargas positivas tentando anular a sonda. Desta forma, a saturação iônica deveria acontecer onde se estabelece o raio óptico, como identificado na Figura 6. A condição de saturação eletrônica demonstra a posição máxima onde existem elétrons disponíveis no arco, mas não suficientes para proporcionar emissão de radiação luminosa significativa.

A técnica do ânodo bi-partido é utilizada para se estabelecer as dimensões na região do ânodo, incluindo a mancha/raiz anódica e a queda de tensão total no ânodo (neste caso 4,5 V).

O raio óptico foi obtido com tratamento das imagens (Figura 7) obtidas com filmadora de alta velocidade e considerandose uma variação de $10 \%$ da intensidade luminosa a partir da referência inicial ('background'), onde as dimensões extraídas seguiram os procedimentos e cuidados estabelecidos na Ref. [11].

Pelos resultados da Figura 6, observa-se que a posição onde ocorre a saturação iônica é semelhante ao raio óptico. Este resultado é coerente, uma vez que a emissão de radiação ocorre pela mudança de estado energético do elétron no íon, ou seja, sua presença é obrigatória e se estende até o valor da saturação iônica. A importância desta região está no fato de que a maioria da corrente elétrica é carregada neste limite.

O maior valor da dimensão do arco é sem dúvida aquele onde seu campo eletromagnético consegue ser 'sentido' por outro condutor, ou seja, até a posição F.C.. De forma intermediária, encontra-se a posição de saturação eletrônica, que é aquela posição onde é possível ainda se definir o estado plasma, com a bainha tendendo a zero. Nesta região de saturação eletrônica ainda há corrente sendo carregada, haja vista que os elétrons carreiam $\sim 97 \%$ do total de corrente. Já a importância da região F.C. é devido à influência do campo eletromagnético do arco sobre sua vizinhança.

Observa-se ainda que a mancha/raiz anódica é menor que o raio óptico encontrado, o que reforça os resultados mostrados anteriormente (Figura 2). Pelo valor obtido $(\sim 2,3 \mathrm{~mm})$, calculase uma densidade de corrente de $\sim 1 \cdot 10^{7} \mathrm{~A} / \mathrm{m}^{2}$, para a corrente de $150 \mathrm{~A}$, ou seja, metade daquela calculada anteriormente para o arco de $100 \mathrm{~A}\left(2 \cdot 10^{7} \mathrm{~A} / \mathrm{m}^{2}\right)$, mas da mesma ordem de grandeza.

Um ponto final a ser discutido refere-se a variação das dimensões do arco em função do seu deslocamento (velocidade de soldagem). Maecker \& Stablein [12] apresentam a Equação 1 como uma relação entre a velocidade de soldagem $T S$ e a curvatura $R$ do arco. Utilizando-se dados obtidos via 


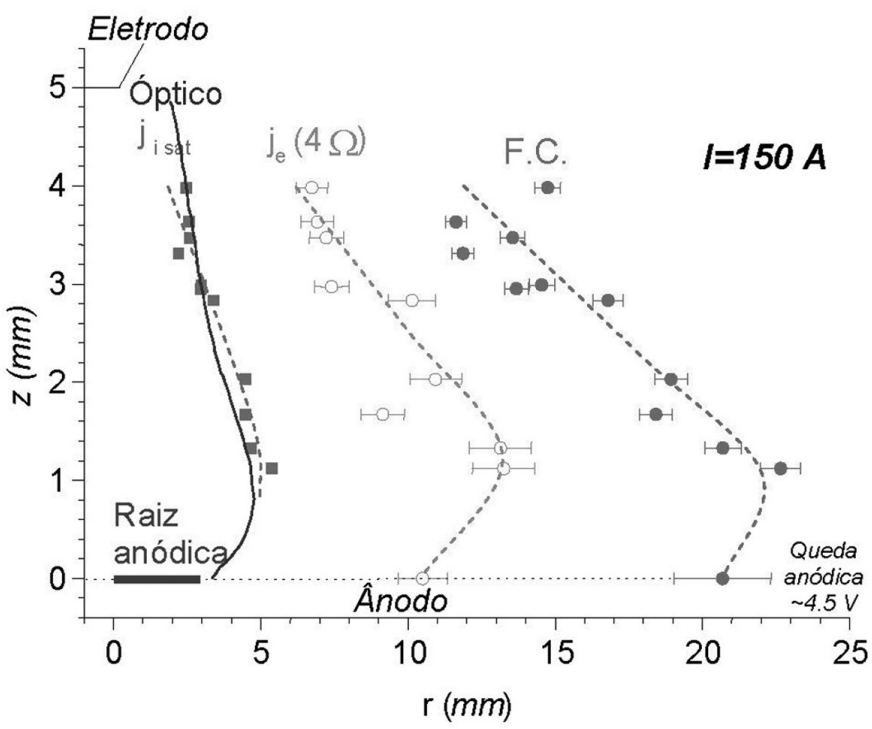

Figura 6. Dimensões do arco para processo TIG, $150 \mathrm{~A}$, argônio puro, comprimento do arco $5 \mathrm{~mm}$, eletrodo $\mathrm{W}+2 \% \mathrm{Th}$ e 3,2 mm de diâmetro, com base no raio óptico, mancha/raiz anódica, saturação iônica $\left(\mathrm{j}_{\text {isat }}\right)$, a saturação eletrônica $\left(\mathrm{j}_{\mathrm{e}}\right)$ e a condição flutuante (F.C.) (compilação dos resultados obtidos na Ref. [3], [9] e [10]).

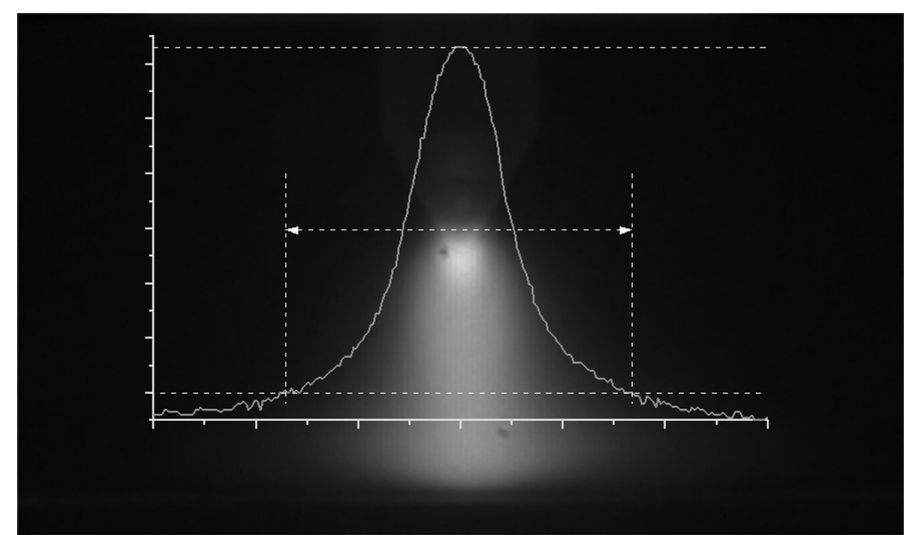

Figura 7. Exemplo de dimensão obtida a partir de imagem retirada da Ref. [3] para arco TIG 150 A, argônio puro, eletrodo $3,2 \mathrm{~mm} \mathrm{~W}+2 \% \mathrm{Th}$.

espectroscopia óptica de emissão [10], mostrados na Tabela 1, é possível obter a Figura 8 para arcos TIG de argônio puro. Isto foi realizado considerando-se $T_{e n v}=300 \mathrm{~K}\left(\rho_{e n v}=1,6224 \mathrm{~kg} / \mathrm{m}^{3}\right), \mathrm{o}$ que preenche os critérios estabelecidos por Maecker \& Stablein, ou seja, $\rho_{\max }<<\rho_{e n v}$. Os valores das propriedades referentes ao argônio puro foram retirados da Ref. [13]. Deve ser ressaltado que a curvatura do arco não é uma função monotônica com a corrente de soldagem, devido à variação das propriedades do plasma com a temperatura do arco.

$T S=5 \cdot \frac{r_{\text {max }}}{r_{\text {env }}} \cdot a_{\text {max }} \cdot \frac{1}{R}$,

Onde, $\alpha_{\max }$ é a difusividade térmica do plasma à máxima temperatura: $\alpha_{\max }=\frac{k_{\max }}{\rho_{\max } \cdot C_{p_{-} \max }}\left[\mathrm{m}^{2} / \mathrm{s}\right]$; $k_{\max }$ é a condutividade térmica do plasma à máxima temperatura observada $[\mathrm{W} / \mathrm{m} \cdot \mathrm{K}]$;

$\rho_{\max }$ é a densidade do plasma à máxima temperatura observada $\left[\mathrm{kg} / \mathrm{m}^{3}\right]$;

$\rho_{e n v}$ is a densidade do plasma à temperatura ambiente $[\mathrm{kg} /$ $\left.\mathrm{m}^{3}\right]$

$C_{p_{\text {max }}}$ é o calor específico à máxima temperatura observada $[\mathrm{J} / \mathrm{kg} \cdot \overline{\mathrm{K}}]$

$T S$ é a velocidade de soldagem;

$R$ é a curvatura do arco.

Tabela 1. Propriedades em função da máxima temperatura obtida por espectroscopia óptica de emissão [10], onde os valores para as propriedades foram retirados da Ref. [13].

\begin{tabular}{c|c|c|c|c}
\hline Corrente [A] & $\begin{array}{c}T_{\max } \\
{[\mathrm{K}][3]}\end{array}$ & $\begin{array}{c}k_{\max } \\
{[\mathrm{W} / \mathrm{m} \cdot \mathrm{K}]}\end{array}$ & $\begin{array}{c}\rho_{\max } \\
{\left[\mathrm{kg} / \mathrm{m}^{3}\right]}\end{array}$ & $\begin{array}{c}C_{p_{\text {max }}} \\
{[\mathrm{J} / \mathrm{kg} \cdot \mathrm{K}]}\end{array}$ \\
\hline 50 & 18000 & 2,3050 & $1,4129 \cdot 10^{-02}$ & 3095,8 \\
\hline 100 & 20000 & 2,6666 & $1,2318 \cdot 10^{-02}$ & 2742,1 \\
\hline 200 & 22000 & 3,1376 & $1,0768 \cdot 10^{-02}$ & 5708,4 \\
\hline
\end{tabular}

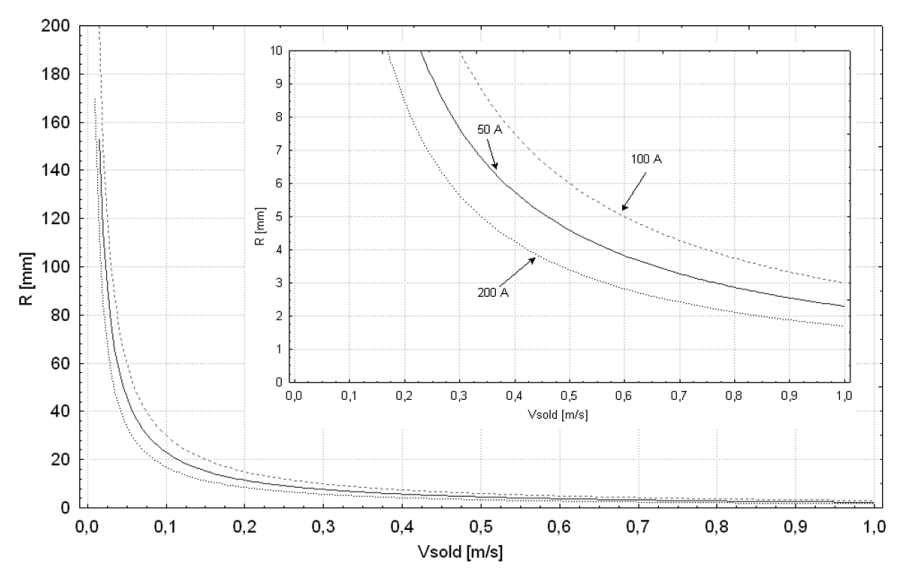

Figura 8. Curvatura do arco em função da velocidade de soldagem (Vsold) para arcos de argônio puro.

De forma a facilitar a visualização do 'dobramento' do arco em função da velocidade do arco, considera-se o valor do seu deslocamento lateral $d$, definido matematicamente na Equação 2, obtida pela análise geométrica da Figura 9. Utilizando-se os dados obtidos na Figura 8, é possível estabelecer a Figura 10. Desta forma, se um deslocamento máximo da ordem de $10 \%$ do total do comprimento do arco for considerado (por exemplo, no caso de $L a=5 \mathrm{~mm}$, o máximo deslocamento seria $0.5 \mathrm{~mm}$ ), então o maior valor possível para a velocidade de soldagem seria entre 0.3 e $0.5 \mathrm{~m} / \mathrm{s}$, o que muito maior que o atualmente em uso na soldagem a arco.

$d=R-0.5 \sqrt{4 R^{2}-L a^{2}}$,

Onde, $\quad d$ é o deslocamento lateral do arco (Figura 9); $R$ é o raio de curvatura do arco (Figura 9); La é o comprimento do arco. 


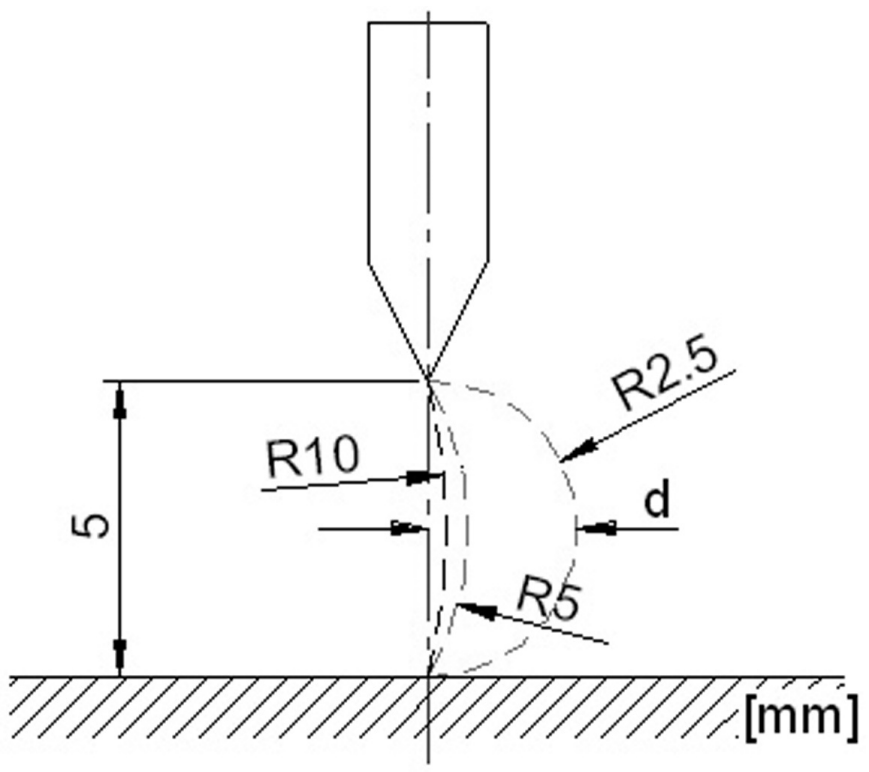

Figura 9. Deslocamento d para diferentes curvaturas em um arco de $5 \mathrm{~mm}$ (eletrodo se movendo para esquerda).

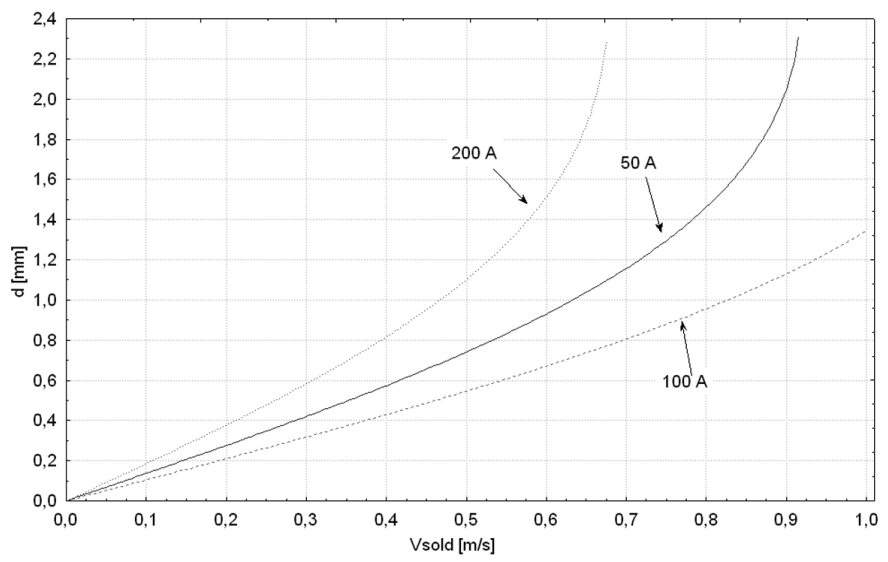

Figura 10. Deslocamento d em função da velocidade de soldagem (Vsold) para um arco de $5 \mathrm{~mm}$.

\section{Observações Finais}

As dimensões do arco foram apresentadas e discutidas com relação ao raio óptico, raio elétrico, posição de saturação iônica e eletrônica, condição flutuante e mancha/raiz anódica. Entendese que estas são as principais características dimensionais de um arco elétrico.

Certamente há a necessidade de se adotar valores arbitrários em certas proposições, como a estimação das dimensões do arco elétrico através das curvas de densidade eletrônica versus temperatura para se estabelecer em que temperatura considerase o nível de ionização para se ter o arco. Entretanto, não se está aqui propondo sua padronização, mas sim uma discussão acerca da mesma, onde sugere-se apontar sempre este valor na divulgação de pesquisas relacionadas.

É possível ainda se estimar as dimensões do arco elétrico através de curvas de pressão e densidade de corrente encontradas na literatura, as quais estariam ainda submetidas à arbitração de valores.

Sem dúvida a principal limitação das características abordadas refere-se ao fato de que os valores aqui mostrados representam o arco elétrico a partir de um processo autógeno. Neste sentido, a continuidade de pesquisas e discussão é mais do que necessária, e o autor convida a comunidade de soldagem para o debate, sugestões e críticas.

\section{Agradecimentos}

$\mathrm{O}$ autor gostaria de agradecer à Capes, à Fapemig, ao $\mathrm{CNPq}$ e ao Laprosolda.

\section{Referências Bibliográficas}

[1] RODRIGUES, C. E. A. L., Avaliação Cinemática $e$ Dinâmica da Transferência Metálica na Soldagem MIG/MAG. 2007, Universidade Federal de Uberlândia. p. 280.

[2] LORD, W. T., The Effective Periphery of a High Pressure Arc. IEEE Trans. Plasma Sci., 1973. 1(4): p. 43-48.

[3] VILARINHO, L. O., Characterisation ofTIGArc Structures Using Experimental Techniques. 2002, Cranfield University. p. 325.

[4] GICK, A. E. F., M. B. C. QUIGLEY, P. H., RICHARDS, T., The Use of Electrostatic Probes to Measure the Temperature Profiles of Welding Arcs. J. Phys. D: Appl. Phys., 1973. 6: p. 1941-1949.

[5] LUDWIG, H. C., Plasma-energy Transfer in Gas-shielded Welding Arcs. Welding Journal, 1959. July: p. 296s-300s.

[6] LANCASTER, J. F., The Physics of Welding. 1986: Pergamon Press \& IIW. 340.

[7] FRANKLIN, R. N., You Cannot Patch Active Plasma and Collisionless Sheath. IEEE Trans. Plasma Sci., 2002. 30(1): p. 352-356.

[8] SCHOECK, P. A., An Investigation of the Anode Energy Balance of High Intensity Arcs in Argon. Modern Developments in Heat Transfer, ed. W.Ibele. 1963: Academic Press. 353-400. [9] VILARINHO, L. O., FANARA, C., Determination of the Anode Voltage Fall in TIG Arcs by Using a Modified Split-Anode Detector. Soldagem \& Inspeção, 2005. 10(4): p. 204-210.

[10] FANARA, C., VILARINHO, L. O., Electrical Characterization of Atmospheric Pressure Arc Plasmas - an Overview. European Physical Journal D, 2004. 28: p. 241-251. [11] VILARINHO, L. O., LUCAS, B., RAGHUNATHAN, S., Cuidados ao se Extrair Dimensões de Fotografia/Filmagem em Soldagem. Soldagem \& Inspeção, 2009. Submetido: 13p.

[12] MAECKER, H. H., STABLEIN, H. G., What Keeps an Arc Standing in a Cross Flow? IEEE Trans. Plasma Sci., 1986. PS14(4): p. 291-299.

[13] MURPHY, A. B., Transport Coefficients of Helium and Argon-Helium Plasmas. IEEE Trans. Plasma Sci., 1997. 25(5): p. 809-814. 